\title{
VALORES TÍPICOS DO "PRODUTO DOSE-ÁREA" (DAP) OBTIDOS DURANTE O ESTUDO VIDEOFLUOROSCÓPICO DA DEGLUTIC̣ÃO*
}

\author{
Milton Melciades Barbosa Costa ${ }^{1,4}$, Lucia Viviana Canevaro ${ }^{2}$, Ana Cecília Pedrosa de Azevedo ${ }^{3}$, \\ Marcelo Diniz Santa Marinha ${ }^{4}$
}

Resumo Analisamos o produto dose-área ("dose area product" - DAP) de 12 pacientes submetidos a videofluoroscopia da deglutição. $O$ objetivo foi estimar a exposição à radiação produzida neste tipo de estudo. Utilizamos medidor de DAP (PTW-Diamentor), que registra, de modo cumulativo, as doses de radiação que atingem o examinado durante todo o procedimento. Obtivemos nossos dados em duas salas dotadas com equipamentos da mesma marca e modelo. 0 protocolo, rigorosamente o mesmo, foi efetuado por um único e experiente profissional. Os valores do DAP para o estudo da deglutição em três fases (oral, faríngea e esofágica) foram: sala 1 (sete pacientes) - $4.101 \mathrm{cGy.cm}{ }^{2}$ de DAP médio com $577 \mathrm{cGy.cm}$ 2/min.; sala 2 (cinco pacientes) - $804 \mathrm{cGy} . \mathrm{cm}^{2}$ de DAP médio com $119 \mathrm{cGy} . \mathrm{cm}^{2} / \mathrm{min}$. Estes resultados díspares foram obtidos de indivíduos com média de 1,57 $\mathrm{m}$ de altura e $56 \mathrm{~kg}$ de peso, em protocolo que se cumpriu em cerca de sete minutos. Concluímos que as doses, cinco vezes mais baixas, obtidas na sala 2, retratam mais adequadamente a exposição determinada pela videofluoroscopia da deglutição. Acreditamos que as doses mais altas, da sala 1, embora dentro dos padrões internacionais para exames do tubo digestivo, devam-se ao desconhecimento do desempenho, nem sempre perfeito, dos equipamentos radiológicos. Esta conclusão encontra apoio no fato de, em nosso meio, não ser usual que os serviços de radiodiagnóstico tenham implementado um rotineiro "programa de garantia de qualidade" e aponta para a importância do DAP na qualificação dos métodos e equipamentos radiológicos.

Unitermos: Dosimetria; Videofluoroscopia; Produto dose-área (DAP); Deglutição.

\begin{abstract}
Typical dose area product (DAP) values for videofluoroscopic swallowing study.
We analyzed the dose area product (DAP) of 12 patients submitted to videofluoroscopic swallowing studies. The aim of this study was to establish, in a preliminary manner, the values of radiation exposure for patients submitted to this type of examination. A Diamentor equipment was used to register the cumulative radiation doses emitted by the x-ray tubes. Two different rooms with identical radiological equipment were used to collect the data. The protocol used was rigorously the same and the same experienced professional performed the examinations. DAP's values for the three phases (oral, pharyngeal and esophagogastric) of the videofluoroscopic swallowing study were, for the room $A\left(7\right.$ patients), 4,101 $\mathrm{cGy} . \mathrm{cm}^{2}, \mathrm{DAP} / \mathrm{min} .=$ $577 \mathrm{cGy} . \mathrm{cm}^{2} / \mathrm{min}$., and for the room B (5 patients), $804 \mathrm{cGy.cm}{ }^{2}, \mathrm{DAP} / \mathrm{min}=119 \mathrm{cGy} . \mathrm{cm}^{2} / \mathrm{min}$. These different results were obtained from patients of the same height and weight, using the same examination time (around 7 minutes) for all patients. We concluded that the data showing doses five times lower in room $B$ are more reliable that those obtained for room $A$ and that DAP and DAP/min. are good elements to inform patient doses imparted by videofluoroscopic swallowing examination. We believe that the high dose differences observed between the two groups are due to lack of calibration of the x-ray equipment, since unfortunately "quality assurance programs" are not routinely implemented.

Key words: Dosimetry; Videofluoroscopy; Dose area product (DAP); Swallowing.
\end{abstract}

\footnotetext{
* Trabalho realizado no Serviço de Radiodiagnóstico do Hospital Universitário Clementino Fraga Filho (HUCFF) da Universidade Federal do Rio de Janeiro (UFRJ), Rio de Janeiro, RJ.

1. Médico, Professor Titular/Doutor do Departamento de Anatomia do Instituto de Ciências Biomédicas da UFRJ.

2. Física Médica, Doutora, do Departamento de Física Médica do Instituto de Radioproteção e Dosimetria da Comissão Nacional de Energia Nuclear (IRD-CNEN).

3. Física Médica, Doutora, da Escola Nacional de Saúde Pública (ENSP) da Fundação Instituto Oswaldo Cruz (Fiocruz).

4. Física Médica, Mestre, do Departamento de Radiologia da Faculdade de Medicina da UFRJ.

Endereço para correspondência: Prof. Dr. Milton M.B. Costa. Laboratório de Motilidade Digestiva/Imagem - sala F1-012, Departamento de Anatomia, Instituto de Ciências Biomédicas. Cidade Universitária, Ilha do Fundão. Rio de Janeiro, RJ, 21949 900. E-mail: mcosta@anato.ufrj.br

Recebido para publicação em 17/5/2002. Aceito, após revisão, em 27/9/2002.
}

INTRODUÇÃO

O objetivo deste estudo foi estabelecer valores preliminares que permitam estimar a exposição à radiação dos indivíduos submetidos à avaliação videofluoroscópica da dinâmica da deglutição.

Exame de excelente qualidade para o estudo da dinâmica das fases oral e faríngea da deglutição, a videofluoroscopia com frequiência é criticada por ser método que utiliza a radiação $\mathrm{X}$ como fonte geradora das imagens. Note-se que usualmente esses mesmos críticos, na defesa da óbvia eficiência de métodos como manometria, pHmetria, faringolaringoendoscopia e mesmo endoscopia digestiva alta, necessitam e admitem o uso da escopia como apoio a seus procedimentos. Assim, quantificar as doses de radiação produzidas pela escopia mostra-se importante não só para a videofluoroscopia, mas também para os demais métodos semióticos empregados para o estudo da dinâmica da deglutição.

A admitida eficiência permitida pelo método videofluoroscópico na observação das fases oral e faríngea ${ }^{(\mathbf{1})}$ estende-se de maneira inquestionável à observação da 
dinâmica e da morfologia esofágica. Observar as três fases da deglutição é essencial para prevenir que não se deixe passar despercebida uma associação ou mesmo causa preponderante no nível do esôfago, que pode estar presente mesmo na ausência de queixas formais. Incluir o estudo da fase esofágica, a despeito do aumento do tempo de exame e, consequientemente, da exposição à radiação $X$, é verdadeiramente importante. Não raro, uma disfagia suspeita como sendo resultante de alterações orofaríngeas deixa ver associação com alguma alteração morfológica ou funcional do esôfago, em especial nos pacientes idosos. Devemos lembrar que pacientes com disfagia de origem baixa podem, por aumento da resistência ao fluxo no esôfago, referir queixas semelhantes às das disfagias de origem alta. É nossa convicção que estes fatos justificam, de modo mais do que suficiente, a inclusão da fase esofágica em protocolos de avaliação videofluoroscópicas das doenças disfágicas. A avaliação esofágica aumenta o tempo de exposição à radiação, mas na relação custo/benefício certamente o benefício prevalece.

A International Comission on Radiological Protection (ICRP) ${ }^{(2,3)}$ conceitua que o exame radiológico clinicamente justificado causa benefício ao paciente e compensa o risco de exposição à radiação a ele associado. No entanto, é importante conhecer as doses de radiação a que os pacientes estarão expostos, para que se possa otimizar a prática do exame.

A radiação interage com o corpo, determinando absorção de energia. A dose fornecida por um determinado exame radiológico distribui-se no corpo, sendo máxima na superfície cutânea da região irradiada. Podemos estimar a radiação na superfície cutânea em um dado momento, usando dosímetros termoluminescentes ("thermoluminescent dosemeters" - TLD), ou a radiação incidente nas áreas expostas, pelo uso de um Diamentor. Este equipamento, acoplado ao tubo de raios $\mathrm{X}$ após o colimador, permite o registro cumulativo da radiação emitida pela ampola, durante o tempo do exame. Mede-se o produto dose-área ("dose area product" - DAP), e mede-se a dose de radiação em relação à área de exposição. Registram-se, cumulativamente, as doses utilizadas. Este tipo de avaliação é particularmente útil para procedimentos que interessam múltiplas regiões do organismo, tal como ocorre na avaliação videofluoroscópica da deglutição, que avalia regiões da cabeça, pescoço e tórax. Variações no tempo de execução do protocolo, nas características das regiões a serem observadas e nas dimensões das áreas expostas são variáveis percebidas e dosadas pelo procedimento.

Estudos preliminares utilizando TLDs, comparando as doses fornecidas pela fluoroscopia (videofluoroscopia), que utiliza intensificador de imagens (sala clara), com as doses fornecidas pela radioscopia clássica (sala escura) e ainda com as doses fornecidas para a obtenção do registro em filmes radiográficos, permitiram a verificação de menor exposição à radiação quando do uso da fluoroscopia, que se vale do intensificador de imagens (sala clara) ${ }^{(\mathbf{4})}$.

O DAP, medido em cGy.cm², mensura toda a radiação emitida pela ampola de raios $\mathrm{X}$ e que atinge o paciente durante a realização do exame. O DAP medido em protocolo, que avaliou as três fases da dinâmica da deglutição, registrou $4.101 \pm$ 881 cGy.cm² $(577 \pm 94 \text { cGy.cm²/min. })^{(5,6)}$, níveis compatíveis com as doses internacionais para exames do tubo digestivo, sendo que na Comunidade Européia foi da ordem de 1.100 cGy.cm² para a esofagografia, de $4.000 \mathrm{cGy} \cdot \mathrm{cm}^{2}$ para a seriografia e de $6.000 \mathrm{cGy} \mathrm{cm}^{2}$ para o clister opaco ${ }^{(7-10)}$. Nesses exames, as radiografias representaram de $20 \%$ a $50 \%$ do DAP $^{(11)}$.

Na videofluoroscopia, que é um exame dinâmico, diferentes regiões com distintas densidades são expostas à radiação. Desse modo, diferentes DAPs são somados. A medida do DAP depende do tipo de equipamento radiológico, do grau de cooperação do paciente, da habilidade do radiologista e do tempo de exposição. Embora definido como dentro dos limites internacionais para exames do sistema digestivo, ampliar o número de observações e conferir a estabilidade das variáveis é necessário.

\section{MATERIAL E MÉTODO}

Os exames videofluoroscópicos foram realizados em duas salas do Serviço de Radiodiagnóstico do Hospital Universitário Clementino Fraga Filho, em ambas utili- zando-se equipamento Medicor modelo UV 56M, tipo FR2, com tubo D19-12/50150 sob a mesa, sistema de TV Videomed 2, com intensificador de imagens RBV 23/ 13, tubo Vidicom padrão 525 linhas, 60 $\mathrm{Hz}$, entrelaçamento $2 / 1$. As imagens foram registradas no formato VHS. Os valores do DAP foram obtidos acoplando-se à saída do tubo de raios X a câmara de ionização e um eletrômetro do Diamentor da PTW modelo M4, na sala 1, e outro com eletrômetro modelo M2, na sala 2. Comparação simples da fidelidade de registro dos Diamentors M4 e M2 foi efetuada e não se determinaram discrepâncias importantes nos registros efetuados pelos dois aparelhos.

Doze indivíduos - sete na sala 1 e cinco na sala 2 - foram analisados quanto às doses de radiação a que ficaram expostos durante o estudo das três fases da deglutição (oral, faríngea e esofagogástrica).

Nas fases oral e faríngea empregou-se o seguinte protocolo de registro videofluoroscópico $^{(9)}$. Registro orofaringolaríngeo em perfil durante inspiração/expiração (a) nasal e (b) oral profundas, e durante (c) fonação. Registro orofaringolaríngeo em póstero-anterior e perfil durante a deglutição: a) de saliva; b) de água; c) de boloteste contrastado de 0,5 a $1,0 \mathrm{~cm}$ de diâmetro somente com ensalivação. Registro orofaringolaríngeo da deglutição a partir da mastigação de bolo-teste contrastado de 1,5 a 2,5 cm de diâmetro, em perfil. Registro orofaringolaríngeo em póstero-anterior e perfil durante a deglutição de bolo líquido contrastado com volumes definidos pelas possibilidades dos examinandos.

A fase esofágica foi registrada em póstero-anterior com deglutições de bolos líquidos (solução de sulfato de bário), com os maiores volumes definidos pela possibilidade dos examinandos em posição ortostática: a) com foco na transição faringoesofágica; b) com acompanhamento no sentido crânio-caudal da migração do bolo da faringe até a transição esofagogástrica; c) em póstero-anterior e oblíquas nos terços médio e inferior do esôfago. Observações em decúbito não foram utilizadas nos dois grupos testados.

A dose de radiação a que os pacientes estiveram expostos (DAP) foi calculada empregando-se produto do DAPm (DAP registrado e expresso pelo Diamentor, em 
cGy.cm²) pelos fatores de correção oferecidos pela PTW para os Diamentors M4 e M2 (fator de correção para tubos de raios $\mathrm{X}$ colocados sob a mesa de exame e o fator de calibração de cada sistema em cada equipamento de raios $\mathrm{X}$ ).

\section{RESULTADOS}

Os valores médios nominais de quilovoltagem $(\mathrm{kV})$ e miliamperagem $(\mathrm{mA})$ obtidos nos exames realizados nos dois aparelhos foram de 65 e 1,2, respectivamente. A altura de $1,57 \mathrm{~m}$ e o peso de $56 \mathrm{~kg}$ foram as médias dos dois grupos. A média do tempo dos exames foi de 7,07 minutos para a sala 1 e de 6,76 minutos para a sala 2. As Tabelas 1 e 2 registram o tempo do exame de cada paciente, o DAP calculado, o DAP/min., que é obtido dividindo-se o DAP calculado pelo tempo despendido no exame, e o DAP normalizado, que é obtido pelo produto da média dos DAP/min (sala $1=577$ e sala $2=119$ ) pelo tempo despendido em cada um dos exames.

\section{DISCUSSÃO}

Quantificar a exposição à radiação determinada por um determinado método, em especial quando ele tem o significado esclarecedor que se observa na avaliação videofluoroscópica da deglutição, é tarefa relevante, porque se tem a oportunidade de definir o real nível de exposição produzido pelo método e se, dentro de valores aceitáveis, corrigir infundadas afirmativas de altas doses, que se sustentam em suposições de profissionais menos informados e no desconhecimento dos leigos.

Doses altas de radiação remontam ao tempo das escopias de "écran" fluorescente (sala escura) ${ }^{(\mathbf{4})}$. As escopias que utilizam intensificadores de imagem (sala clara) mostraram doses compatíveis com os demais exames do sistema digestivo ${ }^{(\mathbf{9 - 1 1})}$.

Que seja do nosso conhecimento, nenhum outro protocolo similar ao que utilizamos para avaliar a dose de radiação a que os pacientes estão submetidos na avaliação videofluoroscópica da deglutição foi desenvolvido, nem para avaliação videofluoroscópica nem radiológica.

Por essa razão, os resultados obtidos com as medidas de DAP em trabalhos anteriores $^{(\mathbf{5 , 6 )}}$ foram propostos para servir como referência e base para futuras avaliações. Era importante tomar em consideração o tipo de protocolo empregado na avaliação da deglutição, quando comparássemos os resultados do DAP. Deveríamos levar em consideração, no protocolo, a in-

Tabela 1 DAPs dos sete pacientes da sala 1 e médias.

\begin{tabular}{|c|c|c|c|c|}
\hline $\begin{array}{c}\text { Paciente } \\
\text { sala 1 }\end{array}$ & $\begin{array}{c}\text { Tempo } \\
(\min .)\end{array}$ & $\begin{array}{c}\text { DAP } \\
(\mathrm{cGy.cm})\end{array}$ & $\begin{array}{c}\text { DAP/min. } \\
(\mathrm{cGy.cm} / \mathrm{min} .)\end{array}$ & $\begin{array}{c}\text { DAP normalizado } \\
(\mathrm{cGy.cm})^{2}\end{array}$ \\
\hline 1 & 7,1 & 3.385 & 477 & 4.096 \\
2 & 7,5 & 5.282 & 704 & 4.372 \\
3 & 7,7 & 4.541 & 590 & 4.442 \\
4 & 6,7 & 4.561 & 680 & 3.865 \\
5 & 7,8 & 4.707 & 603 & 4.500 \\
6 & 5,9 & 2.979 & 505 & 3.404 \\
7 & 6,8 & 3.252 & 478 & 3.923 \\
\hline Médias & 7,07 & 4.101 & 577 & 4.080 \\
\hline
\end{tabular}

Tabela 2 DAPs dos cinco pacientes da sala 2 e médias.

\begin{tabular}{|c|c|c|c|c|}
\hline $\begin{array}{c}\text { Paciente } \\
\text { sala 2 }\end{array}$ & $\begin{array}{c}\text { Tempo } \\
\text { (min.) }\end{array}$ & $\begin{array}{c}\text { DAP } \\
\left(\text { cGy.cm }{ }^{2}\right)\end{array}$ & $\begin{array}{c}\text { DAP/min. } \\
(\text { cGy.cm } 2 / \text { min. })\end{array}$ & $\begin{array}{c}\text { DAP normalizado } \\
\left(\text { cGy.cm }{ }^{2}\right.\end{array}$ \\
\hline 1 & 7,3 & 364 & 50 & 868 \\
2 & 7,0 & 916 & 131 & 833 \\
3 & 6,9 & 1.072 & 155 & 821 \\
4 & 6,8 & 1.179 & 174 & 809 \\
5 & 5,8 & 491 & 85 & 690 \\
\hline Médias & 6,76 & 804,4 & 119 & 804 \\
\hline
\end{tabular}

clusão das três fases da deglutição (oral, faríngea e esofagogástrica) e o tipo de equipamento usado para o registro dos eventos.

Protocolos que não incluíssem as três fases da deglutição poderiam apresentar valores mais baixos de DAP, obtidos pela realização do exame em um tempo mais curto, mas as informações obtidas estariam limitadas pela supressão da observação de importantes eventos. Embora defendamos a realização do exame videofluoroscópico da deglutição com a observação de seus três tempos, é possível reduzir as observações a serem efetuadas nesta etapa esofágica do exame a um simples registro de seu trânsito, após avaliação clínica criteriosa, com vistas a diminuir ainda mais o tempo de exposição à radiação.

A avaliação do DAP por unidade de tempo (DAP/min.) reduz as discrepâncias que poderiam ser causadas pela exclusão ou simplificação de uma fase. Esta exclusão ou simplificação geraria menores valores de DAP, mas valores semelhantes de $\mathrm{DAP} / \mathrm{min}$.

Um DAP normalizado também foi proposto com vistas a se poder estimar qual a presumível dose de exposição de um indivíduo submetido à videofluoroscopia da deglutição. O DAP normalizado representaria a estimativa de dose. Ele é obtido multiplicando-se o DAP/min. pelo efetivo tempo de exposição à radiação $\mathrm{X}$ registrado no cumprimento de um determinado protocolo. Para pacientes de maior compleição que a observada como nossa média $(1,57 \mathrm{~m}$ e $56 \mathrm{~kg})$, se admitiria dose pouco maior, e para pacientes de menor compleição, dose pouco menor, supondo-se equipamentos e metodologia adequados.

Dentro de limites, a média do DAP não muda muito com a compleição física dos indivíduos. As variações dependem, em maior escala, da técnica radiológica empregada, do tempo de exame e da calibração do equipamento. Por esta razão acreditávamos que mesmo com uma amostra pequena tivéssemos definido doses de exposição representativas para o método videofluoroscópico.

Não obstante, ampliar nossa amostra seria fundamental para apurar as médias dos diversos DAPs. Fomos surpreendidos por doses de exposição cinco vezes meno- 
res nos pacientes avaliados na sala 2 do que aqueles observados nos pacientes avaliados na sala 1 .

Tanto os exames da sala 1 quanto os da sala 2 foram feitos pelo mesmo profissional. O tempo médio caiu de 7,07 para 6,78, diferença irrelevante que não justifica variações e nem configura maior apuro na execução de protocolo que se repetiu para todos os pacientes. Técnica, tempo, regime videofluoroscópico e compleição dos pacientes podem ser considerados constantes para os indivíduos dos dois grupos.

As diferenças metodológicas residiram no uso de distintos Diamentors (M4 na sala 1 e M2 na sala 2) e de equipamentos radiológicos semelhantes montados em salas distintas.

Embora os medidores de DAP fossem instrumentos testados e, por isso, com pouca probabilidade de registros tão díspares, uma simples conferência a posteriori foi efetuada comparando-se registros obtidos com os Diamentors M4 e M2. Os instrumentos registraram valores semelhantes.

Os equipamentos radiológicos similares montados em salas distintas foram naturalmente responsabilizados pela significativa diferença de dose cinco vezes maior na sala 1 do que a observada na sala 2 .

Os valores obtidos com a dosagem dos DAPs do nosso primeiro grupo foram comparados com os valores de doses registrados para exames do tubo digestivo em geral e se mostraram dentro da média ${ }^{(\mathbf{7}, \mathbf{8})}$. Mas as doses, cinco vezes menores, registradas nas observações do segundo grupo, atendem com mais realidade a nossa expectativa inicial. Note-se que o regime ra- diológico, nos dois grupos, produziu imagens de boa qualidade e igualmente esclarecedoras.

Embora não tenhamos efetuado aferições técnicas nos equipamentos radiológicos, é de se supor, em razão de termos revisto e aferido todas as demais variáveis, que o aparelho radiológico da sala 1 estivesse com desempenho significativamente inadequado, gerando doses superiores às necessárias e desejadas à obtenção de imagens semióticas. Note-se que o equipamento da sala 1 apresentou piora do seu desempenho, sendo posteriormente desativado por decisão do serviço responsável.

\section{CONCLUSÕES}

Nossas observações permitiram concluir que tanto o DAP quanto o DAP/min. são bons parâmetros para predizer níveis adequados de dose para os exames videofluoroscópicos da dinâmica da deglutição.

Valores típicos de DAP e DAP/min. estão mais próximos dos valores obtidos nos cinco pacientes examinados na sala 2 (804 cGy.cm² e 119 cGy.cm²/min.) do que aqueles observados nos sete pacientes estudados na sala 1 (4.101 cGy.cm² e 557 cGy.cm²/min.).

Nossas observações mostraram ser fundamental a implementação de "programas de garantia da qualidade" nos serviço de radiodiagnóstico e que é nossa responsabilidade dispensar e cobrar permanentes cuidados com os equipamentos em uso.

\section{Agradecimentos}

Agradecemos o suporte, na forma de bolsas e equipamentos, dado pela Fundação Universitária José Bonifácio (FUJB), Fundação de Amparo à Pesquisa do Estado do Rio de Janeiro (Faperj) e Universidade Federal do Rio de Janeiro (UFRJ).

\section{REFERÊNCIAS}

1. Costa MMB. Uso de bolo contrastado sólido, líquido e pastoso no estudo videofluoroscópico da dinâmica da deglutição. Radiol Bras 1996;29:35-9.

2. International Commission on Radiological Protection. ICRP Publication 60. Oxford: Pergamon Press, 1990.

3. International Commission on Radiological Protection. ICRP Publication 73. Oxford: Pergamon Press, 1996.

4. Costa MMB, Nova JLL, Carlos MT, Pereira AA, Koch HA. Videofluoroscopia - um novo método. Radiol Bras 1992;25:11-8.

5. Costa MMB, Canevaro LV, Azevedo ACP. Avaliação dosimétrica do método videofluoroscópico aplicado ao estudo da dinâmica da deglutição. Radiol Bras 2000;33:353-7.

6. Costa MMB, Canevaro LV, Azevedo ACP. Dosimetric assessment of swallowing examinations with videofluoroscopy. International Conference on the Radiological Protection of Patients in Diagnostic and Interventional Radiology, Nuclear Medicine and Radiation Therapy. Malaga, Spain, March 2001.

7. European Commission. Guidance on diagnostic reference levels (DRLs) for medical exposures. Radiation Protection 109. Directorate-General Environment, Nuclear Safety and Civil Protection. European Commission, 1999.

8. European Commission, European Radiation Protection, Education and Training (ERPET). Training course for medical physicists on establishment of reference levels in diagnostic radiology. Passau, Germany, 13-15 September, 1999.

9. Canevaro LV. Avaliação de doses em fluoroscopia gastrointestinal. (Dissertação de Mestrado). Rio de Janeiro: COPPE-UFRJ, 1995.

10. Canevaro LV, Carlos MT, Borges JC, Drexler G, Koch HA. Assessment of doses to patients submitted to fluoroscopic gastrointestinal tract examinations. Rad Prot Dosimetry 1998;80:155-8.

11. Canevaro LV. Otimização da proteção radiológica em fluoroscopia: níveis de referência. (Tese de Doutorado). Rio de Janeiro: UERJ, 2000. 